\title{
MEDICAL AND SURGICAL JOURNAL.
}

Vou.. II.]

TUESDAY, JULY 28, 1829.

[No. 24.

I.

Communicated for the Medical and Surgical Journal.

STRICTURE OF THE RECTUM

In which the Calibre of the Intestine was wholly obliterated.

By Dr. John Stevens, Lic. Mass. and N. Y. Med. Soc.

Mrs. D., of Charlestown, aged 48 years, had, for the most part of her life, enjoyed good health. In July, 1828 , she was taken with pain in the bowels, accompanied with constipation, vomiting, rigors, \&c. The pain was rather unusual, coming on at intervals, and very nearly resembling the pains which take place in the last stage of labor. I made an examination per vaginam, and found the uterus enlarged and apparently pressing hard upon the rectum. She had not menstruated for more than four months, atid was persuaded that the womb was affected. I pushed the uterus backwards and upwards, as far as possible, and requested her to keep in a recumbent posture,-bled her freely, gave her an infusion of senna, and blistered the abdomen. On the fourth day she had an evacuation, which gave partial relief, but the pain still continued very severe. I gave Pil. Hyd. with Extract Conii, a pill every six hours, and she gradually recovered; since

- which time Mrs. D. has suffered much from constipation of the bow- els, having had a similar attack as often as once in cvery five or six weeks, which has lasted from five to eight days, but finally yielded to the same mode of treatment.

June $16 \mathrm{th}, 1829$, I was again called to visit Mrs. D., and found that she had had no fecal evacuation for some days, probably ten or twelve. 'The precise time could not be ascertained. Her pulse were good, and there were no febrile symptoms ; her pains were sovere, and as above described, similar to travail pains, but there was no enlargement of the uterus. She had taken a dose of the Sulph. Magnesia, and also a dose of the Compound Tincture of Aloes, without effect; but after retaining them on the stomach for some hours, had thrown them up. I gave a decoction of senna, manna, and jalap, and enemas of the same.

17th.-No better. Gave Hyd. Subm. gr. x., Pulv. Jalap. gr. xx., and continued enemas.

18th.-No better. Much rumbling in the bowels, as has been usual in every attack. Gave 01 . Ricini et 0l. Terebinth.

19th. - No better. Continued enemas and used warm bath.

20th. - No better. Gave Sulph. Soda, as much as the stomach would bear, through the day.

21st. - No better. Gave pills of Hyd. Subm., Jalap and Colocynth; continued enemas and used friction. 
22d. - No better. I informed Mrs. D.'s friends that I considered her in great danger, and wished for a consultation. They inade choice of Dr. Brown, of Boston. He satv her in consultation this day, and continued to visit her daily with me during the remainder of her life. He entered into a minute examination of her case, and perfectly agreed with me as to its nature and mode of treatment. A large blister was immediately applied to the abdomen, and two ounces of the Infus. Sennæ Comp. directed every two hours, and enemas continued; also the warm bath.

23d.-We saw the patient again. No relief had beeu obtained. Gave Subm. Hyd. et Pulv. Jalap. in large doses ; continued enemas.

24th. - No better. Applied an emollient cataplasm over the whole abdomen, and yave a pill composed of Pulv. Jalap. et Subm. Hyd. et O1. Croton, minim one, every four hours. Continued enemas.

25th. - No better. Pills had been retaincd. Gave an injection of the Infus. Tabaci: it was retained but a short time; it however caused a slight degree of nausea and dizziness. $\Lambda$ so!ution of the Sulph. Soda was given through the day, as the stomach would bear.

26 th. - No better. Directed Hyd. Subm. 3i.- -7 i. to be given every four hours, and enemas continued of senna and salts.

27th.-No better. Gave Ol. Ricini et Ol. 'Terebinth. equal parts, as much as the stomach would bear, and enemas of the same through the day.

28 th. - No better. Much irritation; abdomen somewhat enlarged and tense ; sphincter ani relaxed ; pulse upwards of 100 . Gave enemas of flaxseed tea with Tinct. Opii.
29th.-Worse. Symptoms of inflammation strongly marked; pulse 120 and hard. Bled freely; continued enemas, and gave Tinct. Opii, to procure temporary relief, as the pain was intolerable; gare also an injection of Decoct. Tabaci.

30th.-Considered the case as perfectly hopeless, and recommended a palliative course merely, which was continued until July 2d, when she died in great agony.

Post-mortem Examination.

Previous to her death, being perfectly aware of her situation, she very judiciously and composedly requested me to examine her body, and ascertain the cause of her extreme suffering and death. Accordingly, on the 3d of July, about ten hours after her decease, I proceeded to an examination, assisted by my friend Dr. Sprague. Dr. Brown was also present.-On opening the abdomen, the whole intestiual canal, together with the omentum, was obviously in a state of high inflammation: it was very much distended with flatus and liquids of different kinds, which had been taken ; it also contained, dispersed throughout its whole extent so far as traced, a large quantity of green mucus, blended with feculeut matter in a liquid state. The intestines were carefully dissected from the mesentery, and accurately examined from the stomach donn to the rectum; but no cause of obstruction was met with, until we entered the pelvis. We then discovered a firm stricture of the rectum : the part appeared precisely as though a ligature had been passed tightly round the intestine. On further examination, it was found that the calibre of the rectum was entirely obliterated; a small probe could not be passed through it. This stricture could 
not have been of recent origin, as there were no symptoms of recent inflammation, either about or below it ; in fact, this part of the intestinal canal was the only part free from inflammation.

Query, - was this stricture occasioned by an occasional enlargement of the uterus, or did it a rise from accidental and unknown causes ?

Charlestown, July 13th, 1829.

\section{II.}

Communicated for the Boston Medical and Surgical Journal.

Carotid aneurism, - OPERATION.

Performed at the House of Industry at South Boston,

By WinsLow Lewis, Jr., M.D.

TuE subject of the following operation was a colored female of 26 years of age, married, of very intemperate habits, and of an excessive irritable temperament. Her ordinary occupation was that of a domestic, but her labors were severe, and she was accustomed to carry heavy weights on her head. During an exertion, about five months since, to raise a large body for the purpose of placing it on her head, she thinks she "felt something give way," was immediately faint, and soon after perceived a tumor on the left side of her neck. This increased slowly, and at times caused great pain in the head and eyes, with vertigo; and for some time past she has expectorated a bloody mucus. For the relief of these distressing symptoms, she entered the House of Industry Feb. ruary 25th, 1829. I found, on examination, a tumor of the size of a pigeon's egg on the left side of the neck, under the angle of the jaw, pulsating and exhibiting the usual characteristics of aneurismal disease.
I waited for some weeks, to observe if any effect might be produced on the tumor by the total deprivation of ardent spirits, which must have so much increased the circulating force. I also, during this time, applied over it a moderate compression, and put her upon the lowest diet. 'This was wholly ineffectual as regarded the disease, but her general health became much improved. With Dr. Warren in consultation, the operation was advised, but during the examination to determine the nature of the disease, the necessary pressure on the tumor for the purpose of emptying it, caused such excessive pain and excitement that it could not be effectually done. However, enough could be ascertained to show the true character of the tumor, and to distinguish it from ordinary sivellings in that part; for the tumor did not move with the larynx or trachea, and other indications were evident of aneurism of the common carotid.

Operation, March 9th.-The patient was placed in a chair, with the head inclined to the right and a little bent backwards. The incision was made over that spot where the sterno-cleido-mastoideus muscle crosses the omo-hyoideus, in the direction of the fibres of the tracheal portion of the first named muscle. After the common sheath was exposed, the patient fainted. The operation was completed without difficulty, except that the internal jugular would occasionally interfere with and cover the artery. The patient did not complain of any peculiar sensation on tying the ligature. The pulsation above immediately ceased.-Half an hour after the operation, she suffered from pain on the left side of the head and in the region of the tem- 